Journal of Organometallic Chemistry, 391 (1990) 247-257

Elsevier Sequoia S.A., Lausanne

JOM 20816

\title{
Komplexchemie perhalogenierter Cyclopentadiene und Alkine
}

\section{VI *. Reaktion von Dichlorethin mit $\left(\eta^{5}-\mathrm{C}_{5} \mathrm{H}_{5}\right) \mathrm{Co}(\mathrm{CO})_{2}$. Strukturen von $(\mathrm{CpCo})\left(\eta^{4}-\mathrm{C}_{7} \mathrm{Cl}_{6} \mathrm{O}\right),[\mathrm{CpCo}]_{2}\left[\mu-\left(\eta^{1}, \eta^{2}\right)_{2^{-}}\right.$ $\left.\mathrm{C}_{5} \mathrm{Cl}_{4} \mathrm{O}\right]$ und $\left[\eta^{4}-(\mathrm{CpCo}-\mathrm{CCl}=\mathrm{CCl}-\mathrm{CCl}=\mathrm{CCl})\right](\mathrm{CoCp})$}

\section{Karlheinz Sünkel *}

Institut für Anorganische Chemie der Universität München, Meiserstr.1, D-8000 München 2 (B.R.D.)

(Eingegangen den 13. Februar 1990)

\begin{abstract}
The reaction of $\left(\eta^{5}-\mathrm{C}_{5} \mathrm{H}_{5}\right) \mathrm{Co}(\mathrm{CO})_{2}$ with $\mathrm{ClC} \equiv \mathrm{CCl}$ gives hexachlorobenzene in addition to at least six different products, three of which were characterized by $\mathrm{X}$-ray crystallography: The first $\pi$-complex of hexachlorotropone, $(\mathrm{CpCo})\left(\eta^{4}-\right.$ $\mathrm{C}_{7} \mathrm{Cl}_{6} \mathrm{O}$ ), shows $\eta^{4}$-coordination of the two non-conjugated double bonds, which has not been previously observed in other complexes having cycloheptatrienone ligands. The structure of a 1,2-dicobaltacycloheptadienone is also described for the first time. Finally, the structure of a bimetallic tetrachlorocobaltacyclopentadiene has been verified by XRD methods.
\end{abstract}

\section{Zusammenfassung}

Die Reaktion von $\left(\eta^{5}-\mathrm{C}_{5} \mathrm{H}_{5}\right) \mathrm{Co}(\mathrm{CO})_{2}$ mit $\mathrm{ClC} \equiv \mathrm{CCl}$ ergibt neben Hexachlorbenzol mindestens sechs verschiedene Produkte, von denen drei durch Röntgenstrukturanalyse charakterisiert werden konnten: Der erste $\pi$-Komplex von Hexachlortropon, $(\mathrm{CpCo})\left(\eta^{4}-\mathrm{C}_{7} \mathrm{Cl}_{6} \mathrm{O}\right)$ weist $\eta^{4}$-Koordination zweier nicht benachbarter Doppelbindungen auf, was bisher in anderen Komplexen mit Cycloheptatrienon-Liganden nicht beobachtet wurde. Ebenfalls zum erstenmal wird die Struktur eines 1,2-Dikobaltacycloheptadienons beschrieben. Schließlich konnte die Struktur eines bimetallischen Tetrachlorocobaltacyclopentadienons kristallographisch bestätigt werden.

\section{Einleitumg}

Mit wohl keinem anderen Metallkomplex wurde eine solche Vielzahl von Produkttypen bei der Reaktion mit Alkinen erhalten wie mit $\mathrm{CpCo}(\mathrm{CO})_{2}$ : einfache ein-

\footnotetext{
$\bar{*}$ V. Mitteilung siehe Ref. 1.
} 
und mehrkernige $\pi$-Komplexe, Kobaltole, Cyclobutadien-, $\eta^{4}$-Benzol-, Alkylidinund Cyclopentadienon-Komplexe, Metallacyclobutenone, schließlich sogar Cycloadditionsprodukte des koordinierten Cyclopentadienylrings mit dem Alkin [2-11]. Trotz - oder wegen - seiner hohen Reaktivität [12] ist die Reaktion von Dichlorethin mit Übergangsmetall-Komplexen nur vereinzelt untersucht worden. Dabei zeigte sich jedoch ein durchaus normales Verhalten sowohl gegenüber CarbonylKomplexen [13-15] als auch Metallhalogeniden in hohen Oxidationsstufen [16]. Wir haben kürzlich die Umsetzung mit $\mathrm{Pt}\left(\mathrm{PPh}_{3}\right)_{2}\left(\mathrm{C}_{2} \mathrm{H}_{4}\right)$ beschrieben [17]. In Fortführung dieser Arbeiten berichten wir hier über die Umsetzung von $\mathrm{ClC} \equiv \mathrm{CCl}$ mit $\mathrm{CpCo}(\mathrm{CO})_{2}$.

\section{Ergebnisse und Diskussion}

$\mathrm{ClC} \equiv \mathrm{CCl}$ und $\mathrm{CpCo}(\mathrm{CO})_{2}$ reagieren nach einer gewissen Induktionszeit unter starkem Aufschäumen zu einer schwarzen Masse, aus der nach chromatographischer Aufarbeitung neben $\mathrm{C}_{6} \mathrm{Cl}_{6}$ sechs Reaktionsprodukte isoliert werden konnten. Das Auffinden von Hexachlorbenzol in verschiedenen Frationen der chromatographischen Trennung legt nahe, daß sich dieses als Zersetzungsprodukt verschiedener Komplexe erst auf der Säule bildet. Die spektroskopischen Daten der übrigen Produkte (siehe Tab. 1) zeigen die folgenden Verbindungstypen auf:

(a) Die beiden IR-Banden von 1 zwischen 1600 und $2100 \mathrm{~cm}^{-1}$ weisen auf einen carbonylverbrückten Komplex mit zusätzlicher Ketofunktion hin. Das Auftreten zweier eng benachbarter Cyclopentadienyl-Signale sowohl im ${ }^{1} \mathrm{H}$ - als auch ${ }^{13} \mathrm{C}$ NMR-Spektrum zeigt kleine Unterschiede in der chemischen Umgebung der Kobalt-Atome auf. Ein zweikerniger Komplex mit einer Kobaltacyclobuten-Struktureinheit - wie sie schon früher einmal postuliert wurde [11] - könnte diese spektroskopischen Befunde erklären.

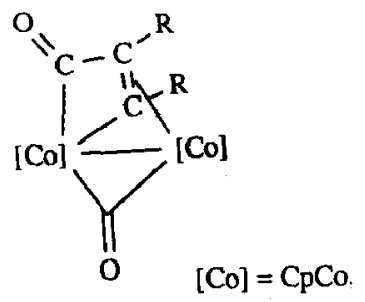

1

Tabelle 1

Spektroskopische Daten von 1-6 ${ }^{\circ}$

\begin{tabular}{llll}
\hline Verbindung & $\operatorname{IR}(\nu(\mathrm{C} \equiv \mathrm{O}, \mathrm{C}=\mathrm{O}, \mathrm{C}=\mathrm{C}))$ & ${ }^{1} \mathrm{H}-\mathrm{NMR}: \delta(\mathrm{CP})$ & ${ }^{13} \mathrm{C}-\mathrm{NMR}: \delta(\mathrm{Cp})$ \\
\hline $\mathbf{1}$ & 1840,1620 & $4.62,4.58$ & $96.7,94.9$ \\
2 & 1645 & 4.49 & \\
3 & 1700 & 4.43 & \\
4 & $2038,1778,1729$ & 4.19 & 89.5 \\
5 & & $4.53,4.56$ & $88.2,88.0$ \\
6 & & $4.68,4.53$ & $86.1,84.4$
\end{tabular}

${ }^{a}$ NMR-Spektren in $C_{6} D_{6}$, IR-Spektren in Nujol. 
(b) Die beiden Komplexe 2 und 3 enthalten nach ihren IR-Spektren ketonische Carbonylgruppen, aber keine terminalen oder verbrückenden CO-Liganden. Die ${ }^{1}$ H-NMR-Spektren mit nur einem Cyclopentadienyl-Signal sprechen für einkernige oder symmetrische zweikernige Komplexe. Durch Röntgenstrukturanalyse konnte 2 als einkerniger Komplex mit Hexachlortropon und 3 als zweikerniges Dimetallacycloheptadienon charakterisiert werden.<smiles>O=C1C(Cl)=C(Cl)C(Cl)=C(Cl)C1Cl</smiles><smiles></smiles>

$2 \quad[\mathrm{Co}]=\mathrm{CpCo} \quad 3$

(c) Die drei IR-Banden zwischen 2100 und $1700 \mathrm{~cm}^{-1}$ im Spektrum von 4 lassen auf eine Struktur mit einem Dichlormaleoyl-Liganden Schließen [18]:<smiles>CC1=C(Cl)C(=O)OC1(C)OC(=O)[O-]</smiles>

(d) 5 besteht möglicherweise aus einem Produktgemisch aus einer Cycloaddition zwischen $\mathrm{ClC} \equiv \mathrm{CCl}$ und $\mathrm{C}_{5} \mathrm{H}_{5}$-Liganden, wie sie schon einmal beschrieben wurde [8]. ${ }^{13} \mathrm{C}-\mathrm{NMR}$-Untersuchungen zeigen sowohl vinylische $\mathrm{C}-\mathrm{Cl}$ - als auch $\mathrm{C}-\mathrm{H}$-Gruppen sowie eine Vielzahl sekundärer $\mathrm{CH}_{2}$ - und tertiärer $\mathrm{CH}$-Gruppen auf; eine eindeutige Zuordnung erscheint nicht möglich. Die Lage einiger ${ }^{13} \mathrm{C}$-Signale läßt auf den Einbau einer oder mehrerer Diethylether-Einheiten schließen. Dies könnte als Folge einer Radikal-induzierten Zersetzung von $\mathrm{ClC} \equiv \mathrm{CCl} \cdot \mathrm{OEt}_{2}$ eingetreten sein [12].

(e) Bei 6 sollte es sich um einen zweikernige Komplex mit verschiedener Umgebung der Kobalt-Atome ohne Metallcarbonyl- oder Ketogruppen handeln; dies konnte auch durch Kristallstrukturanalyse bestätigt werden (siehe unten).<smiles>ClC1=C(Cl)C2(Cl)OC1(Cl)C(Cl)=C2Cl</smiles>

$6[\mathrm{Co}]=\mathrm{CpCo}$. 


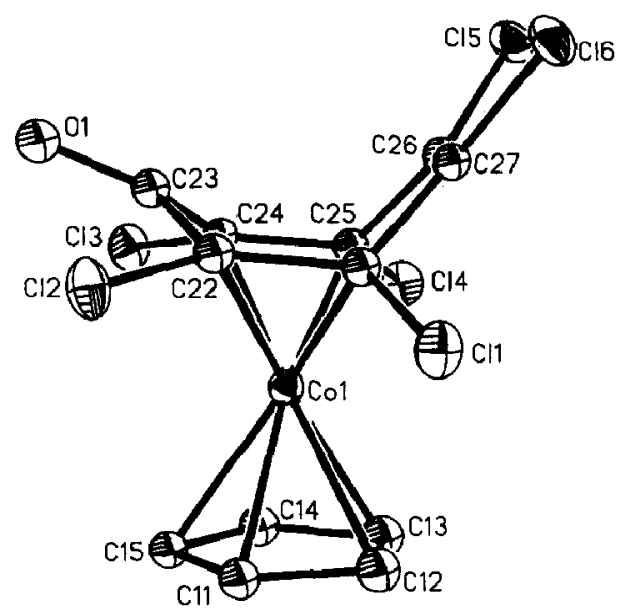

Fig. 1. Struktur von 2 im Kristall (H-Atome weggelassen).

\section{Kristallstrukturanalyse von 2}

Die Struktur von 2 im Kristall ist in Fig. 1 gezeigt. Auffälligstes Merkmal ist die Koordination des Hexachlortropons über zwei nicht benachbarte Doppelbindungen, im Unterschied zu den wenigen bisher bekannten Strukturen von Tropon-Komplexen [19].

Hier liegt nun eher ein Cyclopentadienylkobalt-Komplex mit zwei Monoolefinen vor. Die vier koordinierten C-Atome liegen exakt in einer Ebene, die mit der Cyclopentadienyl-Ringebene einen Winkel von $5.3^{\circ}$ einschließt, also nahezu parallel zu dieser ist. Bedingt durch die zwischen den beiden koordinierten Doppelbindungen liegenden $\mathrm{CO}$ - und $\mathrm{ClC}=\mathrm{CCl}$-Gruppen werden die vier Chlor-Substituenten unter diese Ebene in Richtung auf das Co-Atom gedrückt, wobei der Effekt auf der Carbonylseite erwartungsgemä $B$ mit etwa $31 \mathrm{pm}$ deutlich geringer ist als auf der anderen Seite mit durchschnittlich $51 \mathrm{pm}$. Die C-C-Bindungslängen im Siebenring sind in drei Gruppen unterteilt. Die koordinierten Doppelbindungen sind mit durchschnittlich 141.5(10) pm im üblichen Rahmen und gut $10 \mathrm{pm}$ länger als die unkoordinierte $\mathrm{C}=\mathrm{C}$-Bindung. Ein umgekehrter Trend ist bei den zugehörigen C-Cl-Bindungslängen angedeutet, wenn auch nicht so deutlich ausgeprägt. Die übrigen Bindungslängen entsprechen typischen Einfachbindungen (Tab. 2) [20*].

2 ist unseres Wissens der erste Komplex mit Hexachlortropon, das auch bis vor kurzem schwierig darstellbar war und dessen Reaktivität nur wenig untersucht wurde [21].

\section{Kristallstrukturanalyse von $3[22 *]$}

Wie man Fig. 2 entnehmen kann, handelt es sich bei 3 um ein recht komplexes Molekül. Ein Tetrachlorpentadien-3-on verbruckt die beiden Kobalt-Atome in einer

\footnotetext{
* Die Literaturnummer mit einem Sternchen deutet eine Bemerkung in der Literaturliste an.
} 
Tabelle 2

Wichtige Bindungslängen (pm) und Winkel $\left({ }^{\circ}\right)$ in 2

\begin{tabular}{llllll}
\hline$C(1)-C(21)$ & $203.2(7)$ & $C(1)-C(22)$ & $202.6(8)$ & $C O(1)-C(24)$ & $201.6(7)$ \\
$C(1)-C(25)$ & $201.2(8)$ & $C(21)-C(22)$ & $141.8(9)$ & $C(22)-C(23)$ & $149.1(10)$ \\
$C(23)-C(24)$ & $149.8(12)$ & $C(24)-C(25)$ & $141.0(10)$ & $C(25)-C(26)$ & $151.2(9)$ \\
$C(26)-C(27)$ & $131.0(11)$ & $C(21)-C l(1)$ & $177.2(7)$ & $C(22)-C l(2)$ & $174.9(9)$ \\
$C(23)-O(1)$ & $122.8(9)$ & $C(24)-C(3)$ & $174.4(7)$ & $C(25)-C(4)$ & $176.5(9)$ \\
$C(26)-C((5)$ & $172.5(9)$ & $C(27)-C(6)$ & $172.8(7)$ & & \\
$C(22)-C(23)-C(24)$ & $108.4(6)$ & $C(22)-C(23)-O(1)$ & $124.2(8)$ & $C(24)-C(23)-O(1)$ & $123.9(6)$ \\
$C(25)-C(26)-C(27)$ & $117.0(7)$ & $C(25)-C(26)-C l(5)$ & $119.0(6)$ & $C(27)-C(26)-C l(5)$ & $123.6(6)$ \\
$C(21)-C(27)-C(26)$ & $117.8(6)$ & $C(21)-C(27)-C l(6)$ & $118.8(6)$ & $C(26)-C(27)-C l(6)$ & $123.0(6)$ \\
\hline
\end{tabular}

Weise, daß jedes Co-Atom mit drei C-Atomen dieses Brïckenliganden verbunden ist. Durch die Co-Co-Bindung (241 pm) erhält jedes Co-Atom die 18Elektronenschale. Verbindungen dieses Typs sind als Produkte der Reaktion von $\mathrm{Fe}_{2}(\mathrm{CO})_{9}$ und $\mathrm{Fe}_{3}(\mathrm{CO})_{12}$ mit inneren Alkinen seit langem bekannt [23]; auch einige Cyclopentadienrhodium-derivate $[10 a, 24,25]$ sowie Ruthenium-Komplexe [26] sind beschrieben worden; mit Kobalt sind derartige Verbindungen bisher noch nicht
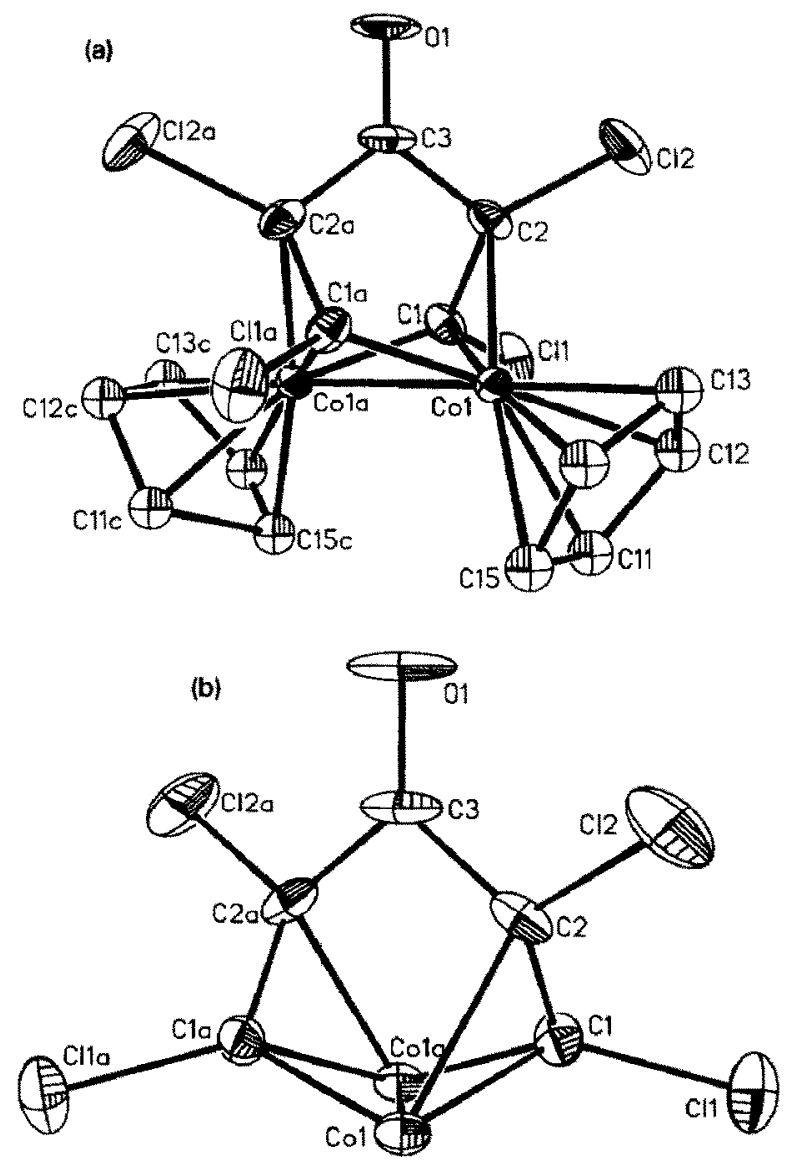

Fig. 2. Zwei Ansichten der Struktur von 3 im Kristall (H-Atome weggelassen): (a) mit (b) ohne Cyclopentadienyl-Ringe. 
Tabelle 3

Wichtige Bindungslängen (pm) und Winkel $\left({ }^{\circ}\right)$ in 3

\begin{tabular}{lllrlr}
\hline $\mathrm{Co}(1)-\mathrm{C}(1)$ & $193.5(11)$ & $\mathrm{Co}(1)-\mathrm{C}(2)$ & $204.6(11)$ & $\mathrm{Co}(1)-\mathrm{C}(1 \mathrm{~A})$ & $187.7(9)$ \\
$\mathrm{Co}(1)-\mathrm{Co}(1 \mathrm{~A})$ & $241.1(4)$ & $\mathrm{C}(1)-\mathrm{C}(2)$ & $142.1(16)$ & $\mathrm{C}(2)-\mathrm{C}(3)$ & $148.5(12)$ \\
$\mathrm{C}(1)-\mathrm{Cl}(1)$ & $174.6(9)$ & $\mathrm{C}(2)-\mathrm{Cl}(2)$ & $174.8(10)$ & $\mathrm{C}(3)-\mathrm{O}(1)$ & $120.1(16)$ \\
$\mathrm{Co}(1)-\mathrm{C}(1)-\mathrm{Cl}(1)$ & $126.5(6)$ & $\mathrm{Co}(1)-\mathrm{C}(1)-\mathrm{C}(2)$ & $73.3(7)$ & $\mathrm{Co}(1)-\mathrm{C}(1)-\mathrm{Co}(1 \mathrm{~A})$ & $78.4(4)$ \\
$\mathrm{Co}(1 \mathrm{~A})-\mathrm{C}(1)-\mathrm{Cl}(1)$ & $126.5(7)$ & $\mathrm{Co}(1 \mathrm{~A})-\mathrm{C}(1)-\mathrm{C}(2)$ & $113.1(6)$ & $\mathrm{Cl}(1)-\mathrm{C}(1)-\mathrm{C}(2)$ & $119.2(7)$ \\
$\mathrm{Co}(1)-\mathrm{C}(2)-\mathrm{Cl}(2)$ & $121.0(6)$ & $\mathrm{Co}(1)-\mathrm{C}(2)-\mathrm{C}(1)$ & $64.9(6)$ & $\mathrm{Co}(1)-\mathrm{C}(2)-\mathrm{C}(3)$ & $107.4(6)$ \\
$\mathrm{C}(3)-\mathrm{C}(2)-\mathrm{Cl}(2)$ & $116.0(7)$ & $\mathrm{C}(3)-\mathrm{C}(2)-\mathrm{C}(1)$ & $112.6(8)$ & $\mathrm{Cl}(2)-\mathrm{C}(2)-\mathrm{C}(1)$ & $124.2(7)$ \\
$\mathrm{C}(2)-\mathrm{C}(3)-\mathrm{O}(1)$ & $125.7(6)$ & $\mathrm{C}(2)-\mathrm{C}(3)-\mathrm{C}(2 \mathrm{~A})$ & $108.7(11)$ & $\mathrm{C}(1)-\mathrm{Co}(1)-\mathrm{C}(1 \mathrm{~A})$ & $90.4(4)$ \\
\hline
\end{tabular}

soukturell charakterisiert worden. In 3 sind die drei Co-C-Bindungen unterschiedlich lang, wobei die beiden Abstände zu den symmetrieäquivalenten $\mathrm{C}(1)$-Atomen noch im Rahmen der üblichen $\mathrm{Co}-\mathrm{C}-\sigma$-Bindungslängen bleiben, während $\mathrm{Co}-\mathrm{C}(2)$ im selben Bereich wie die $\mathrm{Co}-\mathrm{Cp}$-Abstände liegt. Diese Abstände entsprechen den in $(\mathrm{CpRh})_{2}\left[\left(\mathrm{CF}_{3} \mathrm{C}=\mathrm{C}\left(\mathrm{CF}_{3}\right)-\mathrm{CO}-\mathrm{C}\left(\mathrm{CH}_{3}\right)=\mathrm{CCH}_{3}\right)\right]$ gefundenen Werten [24]. Die Bindung $C(1)-C(2)$ ist gleich lang wie die koordinierten Doppelbindungen in 2; dasselbe gilt für die Einfachbindung $\mathrm{C}(2)-\mathrm{C}(3)$ (Tab. 3).

\section{Kristallstrukturanalyse von 6}

Wie man aus Fig. 3 ersieht, handelt es sich hierbei um ein Cobaltol [2]. Eine Metallacyclopentadien-Einheit dient als $\pi$-Ligand für ein weiteres Kobalt-Atom,

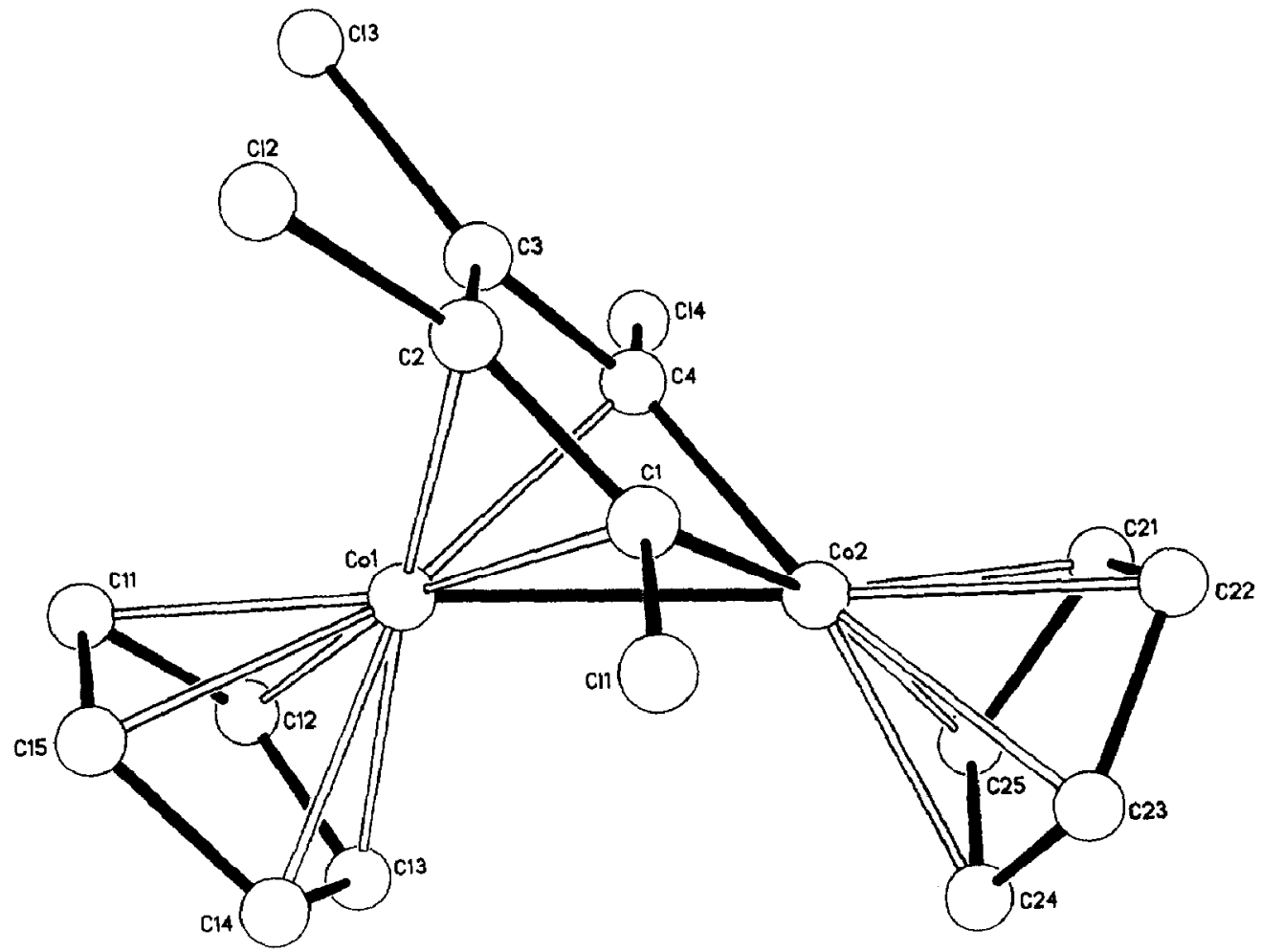

Fig. 3. Struktur von 6 im Kristall (H-Atome weggelassen). 
Tabelle 4

Wichtige Abstände (pm) und Winkel $\left(^{\circ}\right)$ in 6

\begin{tabular}{lcllll}
\hline $\operatorname{Co}(1)-C o(2)$ & $243.0(2)$ & $\mathrm{Co}(1)-\mathrm{C}(1)$ & $208.6(11)$ & $\mathrm{Co}(1)-\mathrm{C}(2)$ & $204.3(12)$ \\
$\mathrm{Co}(1)-\mathrm{C}(3)$ & $204.0(12)$ & $\mathrm{Co}(1)-\mathrm{C}(4)$ & $205.1(12)$ & $\mathrm{Co}(1)-\mathrm{C}(11)$ & $205.0(11)$ \\
$\mathrm{Co}(1)-\mathrm{C}(12)$ & $203.1(13)$ & $\mathrm{C}(1)-\mathrm{C}(13)$ & $201.7(12)$ & $\mathrm{C}(1)-\mathrm{C}(14)$ & $202.6(11)$ \\
$\mathrm{Co}(1)-\mathrm{C}(15)$ & $204.7(10)$ & $\mathrm{Co}(2)-\mathrm{C}(1)$ & $187.1(12)$ & $\mathrm{Co}(2)-\mathrm{C}(4)$ & $185.2(12)$ \\
$\mathrm{C}(1)-\mathrm{Cl}(1)$ & $174.6(12)$ & $\mathrm{C}(2)-\mathrm{Cl}(2)$ & $170.3(12)$ & $\mathrm{C}(3)-\mathrm{C}(3)$ & $172.3(12)$ \\
$\mathrm{C}(4)-\mathrm{Cl}(4)$ & $176.1(12)$ & & & & \\
$\mathrm{C}(1)-\mathrm{Co}(2)-\mathrm{C}(4)$ & $82.3(5)$ & $\mathrm{Co}(2)-\mathrm{C}(1)-\mathrm{C}(2)$ & $118.2(8)$ & $\mathrm{Co}(2)-\mathrm{C}(4)-\mathrm{C}(3)$ & $118.4(9)$ \\
$\mathrm{C}(1)-\mathrm{C}(2)-\mathrm{C}(3)$ & $110.0(10)$ & $\mathrm{C}(2)-\mathrm{C}(3)-\mathrm{C}(4)$ & $111.0(10)$ & & \\
\end{tabular}

das auf diese Weise sandwichartig von zwei Fünfringen, die einen Winkel von $1.1^{\circ}$ miteinander einschließen, koordiniert wird. Dabei sind die Abstände zur $\mathrm{C}_{5} \mathrm{H}_{5}$ Ringebene und der $\mathrm{C}_{4} \mathrm{Cl}_{4}$-Ebene identisch; das zweite Kobalt-Atom und die vier Chlor-Substituenten sind im Mittel mit 6.2 pm nur unwesentlich aus der letzteren Ebene herausgehoben. Der Co-Co-Abstand (243 pm) ist dem in 3 gefundenen praktisch gleich (Tab. 4). Die Abstände von $\mathrm{Co}(2)$ zu den benachbarten C-Atomen im Fünfring entsprechen normalen Co-C- $\sigma$-Bindungen, wie es auch für die kurze Co-C(1)-Bindung in 3 gefunden wurde.

Komplexe wie 3 und 6 werden als Vorstufen für Cyclopentadienon- bzw. Cyclobutadien-Komplexe angesehen [2,23,27], die hier aber nicht nachgewiesen werden konnten. $\mathrm{Ob}$ dies daran liegt, daß sie nicht gebildet wurden, oder bei der Aufarbeitung zersetzt wurden, muß weiteren Untersuchungen überlassen bleiben.

\section{Experimenteller Teil}

Alle Reaktionen wurden unter trockenem Stickstoff in nach den üblichen Verfahren absolutierten und mit Stickstoff gesättigten Lösungsmitteln durchgeführt. Dichlorethin [28] und Dicarbonylcyclopentadienyl-kobalt [29] wurden nach Literaturvorschriften dargestellt. Die chromatographischen Trennungen wurden in einer wassergekühlten Säule von $5 \mathrm{~cm}$ Durchmesser und $100 \mathrm{~cm}$ Länge an Kieselgel 60 (Merck) durchgeführt. Die Kristallstrukturanalysen wurden an einem Syntex-Nicolet-Diffraktometer R3 (Mo- $\boldsymbol{K}_{\alpha}$-Strahlung, Graphit-Monochromator, $\omega$-Scan) durchgeführt (Tab. 5-8). Die zur Struktur-Lösung verwendeten Programme waren SHELXS 76, SHELXTL 4.1 und SHELXTL Plus [30*].

\section{Umsetzung von $\mathrm{CpCo}(\mathrm{CO})_{2}$ mit $\mathrm{ClC} \equiv \mathrm{CCl}$}

$4.02 \mathrm{~g} \mathrm{CpCo}(\mathrm{CO})_{2}(22.3 \mathrm{mmol})$ werden in $40 \mathrm{ml} \mathrm{Et}{ }_{2} \mathrm{O}$ gelöst und mit $6.6 \mathrm{ml}$ einer etherischen Lösung von $\mathrm{ClC} \equiv \mathrm{CCl}$ (ca. $23.1 \mathrm{mmol}$ ) versetzt. Nach etwa $1.5 \mathrm{~h}$ bei Raumtemperatur ist plötzliche starke Gasentwicklung unter Verfärbung nach braunschwarz zu beobachten. Nach 24 h wird abgefrittet und der Niederschlag mit $100 \mathrm{ml} \mathrm{Et}{ }_{2} \mathrm{O}$ extrahiert. Die vereinigten Etherphasen werden bis auf $50 \mathrm{ml}$ eingeengt und dann auf $-78^{\circ} \mathrm{C}$ gekühlt. Nach $4 \mathrm{~d}$ bei dieser Temperatur wird von Niederschlag $\left(\mathrm{C}_{6} \mathrm{Cl}_{6}\right)$ abdekantiert und die Lösung im Vakuum vollständig eingeengt, wobei ein schwarzes Öl zurückbleibt. Dieses wird sechsmal mit je $20 \mathrm{ml}$ Hexan jeweils über Nacht extrahiert. 
Tabelle 5

Kristallographische Angaben zu den Strukturuntersuchungen

\begin{tabular}{|c|c|c|c|}
\hline & 2 & 3 & 6 \\
\hline $\begin{array}{l}\text { Molekiliformel } \\
\text { Molmasse }\end{array}$ & $\begin{array}{l}\mathrm{C}_{12} \mathrm{H}_{5} \mathrm{Cl}_{6} \mathrm{CoO} \\
436.81\end{array}$ & $\begin{array}{l}\mathrm{C}_{15} \mathrm{H}_{10} \mathrm{Cl}_{4} \mathrm{CO}_{2} \mathrm{O} \\
465.91\end{array}$ & $\begin{array}{l}\mathrm{C}_{14} \mathrm{H}_{10} \mathrm{Cl}_{4} \mathrm{Co}_{2} \\
437.89\end{array}$ \\
\hline Kristallgröße (mm) & $0.38 \times 0.35 \times 0.01$ & $0.35 \times 0.40 \times 0.45$ & $0.45 \times 0.35 \times 0.28$ \\
\hline Raumgruppe & $C 2 / c$ & $C 2 / c$ & $\operatorname{Pna2}_{1}$ \\
\hline $\begin{array}{l}a(\mathrm{pm}) \\
b(\mathrm{pm})\end{array}$ & $\begin{array}{l}1459.7(5) \\
737.6(2)\end{array}$ & $\begin{array}{l}1667.3(6) \\
816.8(3)\end{array}$ & $\begin{array}{l}1430.2(4) \\
910.3(3)\end{array}$ \\
\hline$c(\mathrm{pm})$ & 2793.1(11) & $1249.6(3)$ & $1174.7(3)$ \\
\hline$\alpha\left({ }^{\circ}\right)$ & & & \\
\hline $\begin{array}{l}\beta\left({ }^{\circ}\right) \\
\gamma\left({ }^{\circ}\right)\end{array}$ & $107.98(3)$ & $110.45(2)$ & \\
\hline$V\left(\mathrm{pm}^{3}\right)$ & $2.860(2)$ & $1.594(1)$ & $1.5294(7)$ \\
\hline$z$ & 8 & 4 & 4 \\
\hline $\begin{array}{l}\mu\left(\mathrm{Mo}-K_{\mathrm{a}}\right)\left(\mathrm{cm}^{-1}\right) \\
\text { Absorptionskorrektur }\end{array}$ & 23.2 & 27.5 & 28.57 \\
\hline minimale Transmission & 0.115 & 0.068 & 0.082 \\
\hline maximale Transmission & 0.163 & 0.095 & 0.110 \\
\hline gemessene Reflexe & 4403 & 2767 & 1404 \\
\hline 26-Bereich & $4-45^{\circ}$ & $4-50^{\circ}$ & $4-50^{\circ}$ \\
\hline $\begin{array}{l}\text { Indexbereich } \\
\text { davon unabhängig }\end{array}$ & $\begin{array}{l}+h,+k, \pm l \\
1819\end{array}$ & $\begin{array}{l} \pm h,+k,+l \\
1253\end{array}$ & $\begin{array}{l}+h,+k,+l \\
1245\end{array}$ \\
\hline $\begin{array}{l}\text { davon beobachtet } \\
\text { Anzahl verfeinerter }\end{array}$ & 1345 & 896 & 1173 \\
\hline Parameter & 119 & 70 & 92 \\
\hline Lösungsmethode & direkte & direkte & direkte \\
\hline anisotrope Atome & $\mathrm{Co}, \mathrm{Cl}$ & $\mathrm{Co}, \mathrm{Cl}, \mathrm{C}(1-3), \mathrm{O}$ & $\mathrm{Co}, \mathrm{Cl}$ \\
\hline H-Atome berechnet & ja & ja & ja \\
\hline$R$ & 0.0497 & 0.0654 & 0.0544 \\
\hline $\begin{array}{l}R_{\mathrm{w}} \\
\text { maximale Rest- }\end{array}$ & 0.0497 & 0.0653 & 0.0585 \\
\hline elektronendichte & 0.45 & 0.79 & 1.00 \\
\hline
\end{tabular}

Der Rückstand wird in wenig $\mathrm{Et}_{2} \mathrm{O}$ aufgenommen und an Silicagel chromatographiert. Mit Ether eluiert man zunächst eine braunschwarze Bande, die nach Abziehen des Lösungsmittels ein schwarzes Pulver hinterläßt: 1. Dies ist in Benzol sowie polaren Lösungsmitteln wie Methanol, Aceton oder Essigester löslich. Aus Benzol scheidet sich beim Stehen ein Gemisch aus einer glasartigen schwarzen Masse und orangen Kriställchen ab. Gef.: C, 41.90; $\mathrm{H}, 3.20 . \mathrm{C}_{14} \mathrm{H}_{10} \mathrm{Cl}_{2} \mathrm{CO}_{2} \mathrm{O}_{2}$ (399.0) ber.: C, 42.14; H, 2.53\%.

Mit mehr Ether läßt sich von der Säule eine weitere braunschwarze Bande eluieren, die beim Einengen im Vakuum ein ebenfalls schwarzes Pulver hinterläßt. Dieses stellt ein Gemisch von 2 und 3 dar. Beim Stehen einer Benzollösung kristallisieren orange und schwarze Kristalle nebeneinander aus, die mechanisch voneinander getrennt werden können. Bei den ersteren handelt es sich um 2, bei letzteren um 3. Die gesammelten Hexanextrakte werden bis auf etwa $60 \mathrm{ml}$ eingeengt und dann auf $-78^{\circ} \mathrm{C}$ gekühlt. Beim Stehen über Nacht bildet sich ein Niederschlag, der isoliert und wegen seines Gehalts an $\mathrm{C}_{6} \mathrm{Cl}_{6}$ aus Hexan umkristallisiert wird: 4. Es handelt sich um ein gelbes Pulver, das sublimiert werden kann. 
Tabelle 6

Atomkoordinaten $\left(\times 10^{4}\right)$ und isotrope Thermalparameter $\left(\times 10^{-1}\right)\left(\mathrm{pm}^{2}\right)$ von 2

\begin{tabular}{lcccc}
\hline & $x$ & $y$ & $z$ & $U$ \\
\hline$C(1)$ & $1502(1)$ & $11634(1)$ & $1081(1)$ & $31(1)$ \\
$C(11)$ & $2044(7)$ & $13732(11)$ & $744(5)$ & $38(3)$ \\
$C(12)$ & 2173 & 14146 & 1257 & $47(3)$ \\
$C(13)$ & 1251 & 14186 & 1328 & $49(3)$ \\
$C(14)$ & 552 & 13797 & 859 & $44(3)$ \\
$C(15)$ & 1042 & 13517 & 498 & $36(3)$ \\
$C(11 A)$ & $2257(13)$ & $13999(44)$ & $1018(18)$ & $5(10)$ \\
$C(12 A)$ & 1756 & 14203 & 1377 & $3(10)$ \\
$C(13 A)$ & 757 & 13992 & 1123 & $15(11)$ \\
$C(14 A)$ & 640 & 13658 & 607 & $12(11)$ \\
$C(15 A)$ & 1567 & 13662 & 542 & $33(13)$ \\
$C(21)$ & $2650(5)$ & $10033(10)$ & $1424(3)$ & $33(2)$ \\
$C(22)$ & $2203(5)$ & $9456(11)$ & $922(3)$ & $36(2)$ \\
$C(23)$ & $1241(5)$ & $8558(11)$ & $747(3)$ & $35(2)$ \\
$C(24)$ & $574(5)$ & $9538(10)$ & $972(3)$ & $31(2)$ \\
$C(25)$ & $840(5)$ & $10130(10)$ & $1477(3)$ & $35(2)$ \\
$C(26)$ & $1579(5)$ & $9071(10)$ & $1878(3)$ & $36(2)$ \\
$C(27)$ & $2460(5)$ & $9052(10)$ & $1853(3)$ & $32(2)$ \\
O(1) & $969(4)$ & $7585(7)$ & $373(2)$ & $46(1)$ \\
$C l(1)$ & $3826(1)$ & $10956(3)$ & $1574(1)$ & $48(1)$ \\
$C l(2)$ & $2831(1)$ & $9356(3)$ & $481(1)$ & $51(1)$ \\
$C l(3)$ & $-622(1)$ & $9578(3)$ & $589(1)$ & $53(1)$ \\
$C l(4)$ & $-56(1)$ & $11100(3)$ & $1700(1)$ & $49(1)$ \\
$C l(5)$ & $1204(2)$ & $7782(3)$ & $2298(1)$ & $53(1)$ \\
$C l(6)$ & $3366(2)$ & $7738(3)$ & $2243(1)$ & $52(1)$ \\
\hline
\end{tabular}

Tabelle 7

Atomkoordinaten $\left(\times 10^{4}\right)$ und isotrope Thermalparameter $\left(\times 10^{-1}\right)\left(\mathrm{pm}^{2}\right)$ von 3

\begin{tabular}{lllll}
\hline & $x$ & $y$ & $z$ & $U$ \\
\hline$C O(1)$ & $4327(1)$ & $2143(2)$ & $2658(1)$ & $37(1)$ \\
$C l(1)$ & $4092(2)$ & $2010(5)$ & $-65(2)$ & $82(1)$ \\
$C l(2)$ & $3328(2)$ & $-963(4)$ & $1047(3)$ & $90(2)$ \\
$C(1)$ & $4602(6)$ & $1416(13)$ & $1354(8)$ & $46(4)$ \\
$C(2)$ & $4297(6)$ & $22(12)$ & $1774(9)$ & $49(4)$ \\
$C(3)$ & 5000 & $-1038(16)$ & 2500 & $62(8)$ \\
O(1) & 5000 & $-2508(11)$ & 2500 & $108(9)$ \\
$C(11)$ & $3686(9)$ & $4349(16)$ & $2182(11)$ & $53(2)$ \\
$C(12)$ & 3098 & 3024 & 1921 & $53(2)$ \\
$C(13)$ & 3193 & 2186 & 2954 & $53(2)$ \\
$C(14)$ & 3841 & 2993 & 3854 & $53(2)$ \\
$C(15)$ & 4146 & 4329 & 3377 & $53(2)$ \\
$C(11 A)$ & $3352(24)$ & $3770(46)$ & $1863(19)$ & $53(2)$ \\
$C(12 A)$ & 2990 & 2348 & 2167 & $53(2)$ \\
$C(13 A)$ & 3343 & 2173 & 3372 & $53(2)$ \\
$C(14 A)$ & 3922 & 3486 & 3814 & $53(2)$ \\
$C(15 A)$ & 3928 & 4474 & 2881 & $53(2)$ \\
\hline
\end{tabular}


Tabelle 8

Atomkoordinaten $\left(\times 10^{4}\right)$ und isotrope Thermalparameter $\left(\times 10^{-1}\right)\left(\mathrm{pm}^{2}\right)$ von 6

\begin{tabular}{lccll}
\hline & $x$ & $y$ & $z$ & $U$ \\
\hline $\mathrm{Co}(1)$ & $1162(1)$ & $9157(2)$ & 4608 & $35(1)^{a}$ \\
$\mathrm{Co}(2)$ & $864(1)$ & $11604(2)$ & $5351(2)$ & $38(1)^{a}$ \\
$\mathrm{C}(1)$ & $1530(8)$ & $10157(13)$ & $6141(10)$ & $39(3)$ \\
$\mathrm{Cl}(1)$ & $2670(2)$ & $10272(4)$ & $6663(4)$ & $66(1)^{a}$ \\
$\mathrm{C}(2)$ & $1091(8)$ & $8770(12)$ & $6318(10)$ & $35(3)$ \\
$\mathrm{Cl}(2)$ & $1507(3)$ & $7268(4)$ & $7020(3)$ & $63(1)^{a}$ \\
$\mathrm{C}(3)$ & $165(8)$ & $8769(12)$ & $5813(11)$ & $44(3)$ \\
$\mathrm{Cl}(3)$ & $-589(2)$ & $7295(4)$ & $5886(4)$ & $68(1)^{a}$ \\
$\mathrm{C}(4)$ & $-20(8)$ & $10122(13)$ & $5254(11)$ & $40(3)$ \\
$C l(4)$ & $1087(2)$ & $10266(4)$ & $4514(5)$ & $68(1)^{a}$ \\
$C(11)$ & $1206(7)$ & $7368(10)$ & $3549(11)$ & $74(5)$ \\
$C(12)$ & 894 & 8637 & 2958 & $60(4)$ \\
$C(13)$ & 1604 & 9722 & 3038 & $63(4)$ \\
$C(14)$ & 2355 & 9124 & 3678 & $83(5)$ \\
$C(15)$ & 2109 & 7669 & 3995 & $75(4)$ \\
$C(21)$ & $81(5)$ & $13466(12)$ & $5454(11)$ & $77(4)$ \\
$C(22)$ & 806 & 13531 & 6277 & $74(5)$ \\
$C(23)$ & 1675 & 13457 & 5694 & $66(4)$ \\
$C(24)$ & 1486 & 13346 & 4511 & $73(4)$ \\
$C(25)$ & 501 & 13351 & 4362 & $81(5)$ \\
\hline
\end{tabular}

${ }^{a}$ Äquivalente isotrope $U$ berechnet als ein Drittel der Spur des orthogonalen $U_{t f}$-Tensors.

Die abdekantierte Lösung wird fast vollständig eingeengt und an $\mathrm{Al}_{2} \mathrm{O}_{3}$ chromatographiert $(3 \times 10 \mathrm{~cm}$, Hexan stationär). Zunächst wird mit reinem Hexan, dann unter Zugabe von $\mathrm{Et}_{2} \mathrm{O}$ bis zu einem Verhältnis 1/1 eine orangegelbe Bande eluiert, dann mit zunehmendem $\mathrm{Et}_{2} \mathrm{O}$-Gehalt bis $100 \%$ eine dunkelrotbraune Zone. Erstere liefert beim Einengen im Vakuum ein hellgelbes Pulver, 5. Letztere ergibt fast schwarze Kristalle, 6. Gef. C, 39.56; $\mathrm{H}, 2.72 . \mathrm{C}_{14} \mathrm{H}_{10} \mathrm{Cl}_{4} \mathrm{Co}_{2}$ (437.9) ber.: C, 38.40; $\mathrm{H}, 2.30 \%$.

Der Rest auf der Saule läßt sich auch nicht mit polaren Lösungsmitteln wie THF oder Aceton eluieren.

\section{Dank}

Mein besonderer Dankt gilt Herrn Prof. Dr. W. Beck für seine wohlwollende Unterstützung mit Institutsmitteln.

\section{Literatur}

1 K. Sunkel, D. Steiner, J. Organomet. Chem., 368 (1989) 67.

2 M.D. Rausch, R.A. Genetti, J. Org. Chem., 35 (1970) 3888.

3 W.-S. Lee. H.H. Brintzinger, J. Organomet. Chem., 127 (1977) 93.

4 G.A. Ville, K.P.C. Vollhardt, M.J. Winter, Organometallics, 3 (1984) 1177.

5 H. Sakurai, J. Hayashi, J. Organomet. Chem., 70 (1974) 85.

6 R.S. Dickson, G.R. Tailby, Aust. J. Chem., 23 (1970) 1531.

7 B. Eaton, J.M.O'Connor, K.P.C. Vollhardt, Organometallics, 5 (1986) 394.

8 M.B. Gomes de Lima, J.E. Guerchais, F.J. LeFloch-Perennou, P.L'Haridon, F.Y. Petillon, J. Talarmin, Organometallics, 6 (1987) 1930.

9 F. Serratosa, Acc. Chem. Res., 16 (1981) 170, und dort zitierte Literatur. 
10 (a) P.A. Corrigan, R.S. Dickson, G.D. Fallon, L.J. Michel, C. Mok, Aust. J. Chem., 31 (1978) 1937; (b) U. Kölle, B. Fuss, Chem. Ber., 119 (1986) 116.

11 (a) W.A. Herrmann, C. Bauer, J. Weichmann, J. Organomet. Chem. 243 (1983), C21; (b) R.S. Dickson, G.S. Evans, G.D. Fallon, Aust. J. Chem. 38 (1985) 273.

12 S.Y. Delavarenne, H.G. Viehe in H.G. Viehe (Ed.), The Chemistry of Acetylenes, Marcel Dekker, New York, 1969, S.651.

13 C.G. Krespan, J. Org. Chem., 40 (1975) 261.

14 G. Varadi, G. Palyi, Inorg. Chim. Acta, 20 (1976) L33.

15 N.A. Ustynyuk, V.N. Vinogradova, D.N. Kravtsov, Organomet. Chem. USSR, 1 (1988) 45.

16 K. Stahl, F. Weller, K. Dehnicke, Z. Anorg. Allg. Chem., 533 (1986) 73.

17 K. Süakel, J. Organomet. Chem., 348 (1988) C12.

18 R.S. Dickson, H.P. Kirsch, Aust. J. Chem., 27 (1974) 61.

19 D.P. Dodge, J. Am. Chem. Soc., 86 (1964) 5429; D.L. Smith, L.F. Dahl, ibid., 84 (1962) 1743; J. Klimes, E. Weiss, Chem. Ber., 115 (1982) 2175.

20 Der Cyclopentadienylring zeigt eine Fehlordnung im Kristall, die nicht richtig aufgelöst werden konnte, was aber zur Interpretation der Struktur nicht von Bedeutung ist.

21 S. Drüecke, P. Imming, T. Kämpchen, G. Seitz, Chem. Ber., 121 (1988) 1595.

22 Bedingt durch die Lage der zentralen Carbonylgruppe auf einer zweizähligen Drehachse wird eine Molekülhälfte aus der anderen durch eine $C(2)$-Operation generiert. Da das reale Molekül sich aber nicht so ideal verhălt, entsteht eine Unordnung im Cyclopentadienyl-Liganden, die sich nur schwierig behandeln lieB. Wir haben deshalb den Fünfring als starres regelmäßiges Fünfock mit errechneten Wasserstoffpositionen und mit gemeinsamen Temperaturfaktoren fixiert und dann die Belegungsfaktoren und $U$-Werte verfeinert. Trotz dieses etwas willkürlichen Vorgehens glauben wir, daß sich dies auf die Ergebnisse im eigentlich interessierenden Teil des Moleküls nicht oder nur geringfügig auswirkt.

23 W. Hubel in I. Wender, P. Pino (Eds.), Organic Synthesis via Metal Carbonyls, Vol. I, Wiley-Interscience, 1968, S.273.

24 R.S. Dickson, F.I. McClure, R.J. Nesbit, J. Organomet. Chem. 349 (1988) 413.

25 (a) P.A. Corrigan, R.S. Dickson, S.H. Johnson, G.N. Pain, M. Yeoh, Aust. J. Chem., 35 (1982) 2203; R.S. Dickson, B.M. Gatehouse, S.H. Johnson, Acta Cryst. B, 33 (1977) 319.

26 F. Muller, G.V. Koten, L.H. Palm, K. Vrieze, M.C. Zoutberg, D. Hejdenrijk, E. Kragten, C.H. Stam, Organometallics, 8 (1989) 1340.

27 B. Eaton, J.M.O'Connor, K.P.C. Vollhardt, Organometallics, 5 (1986) 394.

28 J. Pielichowski, R. Popielarz, Synthesis, (1984) 433.

29 W.P. Fehlhammer, K. Öfele, W.A. Herrmann in G. Brauer (Ed.), Handbuch der Präparativen Anorganischen Chemie, Bd. 3, 3. Aufl., Ferdinand Enke Verlag, Stuttgart, 1981, S.1876.

30 Weitere Einzelheiten zu den Kristallstrukturuntersuchungen können beim Fachinformationszentrum Karlsruhe, Gesellschaft fïr wissenschaftlich-technische Informationen mbH, D-7514 EggensteinLeopoldshafen 2, unter Angabe der Hinterlegungsnummer CSD-54472, der Autorennamen und des Zeitschriftenzitats angefordert werden. 